Revue

Revue de l'histoire des religions

de Ihistoire des religions

La crisi modernista nella cultura europea : atti del Convegno di studi, Giacomo Losito (a cura di)

Roma, Istituto della Enciclopedia italiana (« Biblioteca dell’Enciclopedia. Scienze e filosofia »), 2012

Louis-Pierre Sardella

\title{
OpenEdition
}

Journals

Édition électronique

URL : https://journals.openedition.org/rhr/8452

DOI : $10.4000 /$ rhr.8452

ISSN : 2105-2573

Éditeur

Armand Colin

Édition imprimée

Date de publication : 1 septembre 2015

Pagination : 467-469

ISBN : 978-2-200-92993-0

ISSN : 0035-1423

Référence électronique

Louis-Pierre Sardella, «La crisi modernista nella cultura europea : atti del Convegno di studi, Giacomo Losıтo (a cura di) », Revue de l'histoire des religions [En ligne], 3 | 2015, mis en ligne le 21 octobre 2015, consulté le 07 décembre 2022. URL : http://journals.openedition.org/rhr/8452 ; DOI : https://doi.org/ 10.4000/rhr.8452

Ce document a été généré automatiquement le 29 septembre 2020.

Tous droits réservés 


\section{La crisi modernista nella cultura europea : atti del Convegno di studi, Giacomo LOSITo (a cura di)}

Roma, Istituto della Enciclopedia italiana (« Biblioteca dell’Enciclopedia. Scienze e filosofia »), 2012

\section{Louis-Pierre Sardella}

\section{RÉFÉRENCE}

La crisi modernista nella cultura europea : atti del Convegno di studi, Giacomo Losito (a cura di), Roma, Istituto della Enciclopedia italiana («Biblioteca dell'Enciclopedia. Scienze e filosofia »), 2012, 385 p., 25 cm, $35 €$, ISBN 978-8-8120-0169-9.

1 Giacomo Losito était particulièrement qualifié pour rassembler, introduire et publier les communications faites lors du colloque organisé à Rome en avril 2005 sur la crise moderniste dans la culture européenne.

Disons-le tout de suite : le contenu de l'ouvrage ne répond pas tout à fait au titre puisque l'Europe dont il est question se limite - à l'exception d'une communication sur l'Espagne - à l'Italie (Saint-Siège inclus) et à la France. Certes, il s'agit des deux pays dans lesquels la crise moderniste a connu les plus grands retentissements, mais la Grande-Bretagne n'y a pas été étrangère (Hügel, Tyrrell) ni d'une certaine façon la Belgique (intervention du cardinal Mercier). Cette géographie limitée reflète l'état de la recherche sur le modernisme, particulièrement active en Italie et actuellement centrée sur les positions romaines comme en témoigne la publication récente d'archives de la Congrégation pour la défense de la foi concernant la mise à l'Index des livres de Loisy et l'élaboration du décret Lamentabili par Claus Arnold et Giacomo Losito. Ceci dit, l'intérêt des dix-sept contributions réunies est indéniable et leur apport à une meilleure connaissance du phénomène moderniste incontestable. 
3 La première partie, consacrée au "point de vue de Rome », revient sur l'origine de la crise moderniste ainsi que sur l'encyclique Pascendi et sa réception. La deuxième partie présente quelques thèmes du débat moderniste en France (cinq des six contributions la concernent - une sur Loisy, trois sur Blondel, la dernière sur Marrou) et en Espagne. La troisième partie enfin est centrée sur quelques grandes figures du modernisme en Italie. Il n'est pas possible, dans le cadre de cette recension, de rendre compte de chacune des communications. De leur ensemble se dégagent quelques points forts qui sont autant de jalons pour une compréhension plus fine de ce moment capital de l'histoire intellectuelle de l'Église contemporaine.

4 La crise moderniste ne peut pas être réduite à une classique querelle des Anciens et des Modernes. Une lecture attentive de l'encyclique Pascendi met en relief le fait que, aux yeux de ses rédacteurs, le modernisme touche à l'interprétation du discours théologique et à l'exposition de la rationalité de la foi à l'heure où la méthode historicocritique entre en conflit avec la méthode scolastique, sans que l'on dispose - et c'est en effet le point capital qui est très bien analysé par Paul Guilbert ( «Le origini del modernismo ») - " des médiations épistémologiques entre le divin et l'humain ». C'est sur ce point que les réflexions de Blondel sont essentielles. Dans la controverse qui l'oppose à l'abbé Vénard en 1904-1905 et qu'analyse Giacomo Losito (" De la valeur historique du dogme»), Blondel démonte les différentes acceptions de la notion de «fait » et renvoie dos à dos historicisme et extrinsécisme en recourant à la notion de tradition (ce qui «fait passer quelque chose de l'implicite perçu à l'explicite connu ») à la fois proche et différente de la notion de développement dogmatique élaborée par Newman comme le montre Peter Reifenberg («Sviluppo e tradizione. Dinamica vitale delle movenza e del movimento permanente : Newman e Blondel »).

5 Pour en revenir au point de vue romain, sa compréhension suppose de replacer la condamnation du modernisme en relation avec le projet idéologico-religieux du pape Sarto clairement revendiqué dans sa devise : Instaurare omnia in Christo et sa mise en œuvre dans la réforme du droit canon ainsi que dans les réformes concernant la liturgie, la pastorale, la catéchèse... Pie X, dans cette perspective, apparaît comme un « rénovateur conservateur». Ainsi en n'isolant pas la controverse moderniste des aspects juridiques, politiques et sociaux de l'affrontement entre l'Église et la modernité, il est possible de dépasser l'opposition simplificatrice entre catholicisme intransigeant intégriste et catholicisme libéral moderniste, et de considérer - au moins à titre d'hypothèse - que les critères discriminants entre les différentes positions à l'intérieur de l'Église ne sont pas tant liés à la controverse doctrinale, qu'à la différence de stratégie pour répondre au défi plus global de la place de l'Église dans le monde moderne (Carlo Fantappiè, « Il codex iuris canonici del 1917 e la modernita giuridica della Chiesa »).

6 Toutefois, le défi majeur aux yeux de Loisy, principal protagoniste de la crise, au-delà de la controverse avec Harnack, était de "réconcilier la science ramenée à ses justes limites avec la foi libérée d'une expression théologique caduque ». Tout en posant la séparation de la science et de la théologie, du monde et de Dieu, il utilise le concept de développement, que Newman appliquait au dogme, à l'ensemble de la révélation au risque de la faire apparaître comme un processus immanent. En se fondant sur le grand manuscrit (désormais publié) qui a servi de carrière aux "petits livres rouges ", le regretté François Laplanche ("Loisy fra le scienze profane e le scienze sacre ») estime que l'incompréhension dont Loisy a été victime réside dans l'imprécision des concepts 
qu'il utilisait, comme par exemple celui de symbole, et dans le fait que l'on n'a pas assez fait attention à la place, certes discrète dans ses livres, mais bien réelle, qu'il accordait au magistère pontifical comme contrepoids nécessaire à l'évolution incessante de la tradition vivante.

7 La troisième partie permet d'approfondir la spécificité du modernisme italien dans l'arbre généalogique duquel il faut faire une place à Rosmini, dont la pensée, non pas tant dans sa dimension théorique que dans sa dimension spirituelle et réformatrice celle des Cinq plaies de l'Église - a notablement influencé des modernistes italiens. C'est ainsi que Paolo Marangon ("L'eredita di Rosmini in Fogazzaro e Semeria ») montre comment A. Fogazzaro philo-rosmanien en vient, sous l'influence de G. Semeria, à adhérer au projet apologétique de Blondel et de Laberthonnière. L'étude des deux principales figures que sont Romolo Murri et Ernesto Buonaiuti (Rocco Cerrato, " Buonaiuti, Murri e la crisi modernista ») met en lumière l'importance de la problématique politico-sociale du modernisme italien ainsi que l'intensité des débats intellectuels dans la péninsule, en particulier celui induit par Giovanni Gentile qui critique l'esprit de compromission des modernistes et qui considère que leurs positions sont incohérentes dans leur volonté de concilier leur adhésion aux principes de la science moderne et leur fidélité au dogme. (Marcello Mustè, "Il "modernismo" di giovanni Gentile"; Mauro Visentin, « La posizione di Gentile di fronte al modernismo »).

8 Au-delà des controverses intellectuelles qui n'ont sans doute touché qu'un cercle restreint de catholiques, on doit s'interroger sur la diffusion beaucoup plus large d'attitudes et de comportements qui ont modifié plus en profondeur le rapport des fidèles à l'Église. C'est ce que nous donne à voir Cettina Militella («Figure femminili del modernismo. Un tentativo di riletture teologica») en faisant le portrait de quatre femmes d'origine sociale différente, plus ou moins liées aux cercles modernistes de Rome ou de Milan, mais toutes militantes féministes. Leur « modernisme " pratique se caractérise par un fort engagement social, une aspiration à une réforme de l'Église qui la rende plus évangélique, une volonté de développer une participation éclairée des fidèles à la liturgie, un souci d'œcuménisme. On ne peut qu'être frappé par la lucidité de leur diagnostic et l'audace de leur prise de parole qui leur valurent d'ailleurs des mises à l'Index et, pour deux d'entre elles, l'exclusion des sacrements. Est-il abusif de les dire modernistes au motif qu'elles n'ont pas été étiquetées ainsi ni de leur vivant ni jusqu'ici par l'histoire? Non, si l'on admet que la racine du modernisme réside dans l'affirmation de la primauté de la conscience sur l'autorité doctrinale. Rien n'est plus significatif de cette revendication d'autonomie dans l'appréciation des actes du magistère que la déclaration de l'une d'entre elles: "J'obéis en tout à l'autorité ecclésiastique, excepté dans son antiféminisme, car celui-ci est contraire aux lois humaines et divines. »

9 Cet ouvrage, on le voit, offre un ample panorama sur la crise moderniste, et à cet égard il prend place parmi les ouvrages de référence (même si l'on peut regretter l'absence d'index) sur ce moment "matrice intellectuelle du catholicisme contemporain" (Étienne Fouilloux) durant lequel se sont posées, au-delà de la critique historique, audelà du problème du développement du dogme, au-delà finalement de la conciliation d'une conception de l'Église figée sur le semper eadem avec celle d'une Église ouverte au semper nova, des questions tout aussi importantes d'un point de vue pratique, car touchant l'ensemble des fidèles, celles qui provenaient de l'inadéquation du discours officiel de l'Église avec les exigences des consciences modernes. 